\title{
Comunicação
}

[Communication]

\section{Avaliação da qualidade microbiológica do soro de queijos Minas padrão e mozarela produzidos em quatro regiões do estado de Minas Gerais}

\author{
[Microbiological profile of the whey of "Minas padrão" and mozzarella cheese produced in \\ Minas Gerais state (Brazil)] \\ L.V. Teixeira ${ }^{1}$; L.M. Fonseca ${ }^{2 *}$, L.D.M. Menezes ${ }^{3}$ \\ ${ }^{1}$ Aluna de pós-graduação - EVUFMG \\ ${ }^{2}$ Escola de Veterinária - UFMG \\ Caixa Postal 567 \\ 30123-970 - Belo Horizonte, MG \\ ${ }^{3}$ Instituto Mineiro de Agropecuária - Belo Horizonte, MG
}

A indústria queijeira representa um importante segmento do setor lácteo. Minas Gerais é o maior produtor de queijo do Brasil, produzindo $215 \mathrm{mil}$ toneladas por ano, o que equivale a $50 \%$ da produção nacional (Martins, 2001). Em Minas Gerais, os mais produzidos são, em ordem, o queijo mozarela, o queijo-de-minas (Minas padrão e Minas frescal) e o requeijão, correspondendo, respectivamente, a $24 \%, 21 \%$ e $14,5 \%$ do total da produção. A maior parte dos laticínios do estado está concentrada nas regiões Sul de Minas (36,5\%), Zona da Mata (17\%), Triângulo Mineiro e Alto Paranaíba $(14,8 \%)$ e Zona Metalúrgica $(14,4 \%)$ (Martins, 2001). O soro lácteo pode ser definido como a fração aquosa do leite que é separada da caseína durante a produção de queijos, correspondendo a cerca de $90 \%$ do volume do leite, levando consigo 50 a $55 \%$ dos sólidos totais do mesmo (Kosikowski, 1979; Furtado e Lourenço Neto, 1994).

A utilização do soro na indústria alimentícia vem sendo estudada por diversos autores, e cada vez mais tem-se utilizado essa matéria-prima na elaboração de novos produtos, seja como simples substituto da água ou como ingrediente de funcionalidade reológica ou nutricional. Esses novos produtos passaram, então, a fazer parte da dieta da população, incluindo os idosos e principalmente as crianças, o que faz com que o controle da qualidade, não só nutricional, mas também higiênico-sanitário do soro, seja fundamental (Chiappini et al., 1995a,b).

Poucos são os trabalhos que avaliaram a microbiota do soro. Quanto à qualidade microbiológica, o soro pode ser um produto de curta vida útil devido ao elevado valor nutritivo, às condições de umidade $\mathrm{e}$ ao $\mathrm{pH}$, que são favoráveis ao crescimento microbiano. Alguns trabalhos demonstraram que é preocupante a contaminação por coliformes (Chiappini et al., 1995a).

Não existem na legislação brasileira atual padrões para sua inspeção físico-química ou microbiológica. Dentre os produtos que aguardam a criação dos padrões de identidade e qualidade estão o Minas padrão e a ricota, que são produtos de origem e destino do soro de queijo, respectivamente (Teixeira, 2005).

Para se fazer uma avaliação da qualidade microbiológica do soro de queijos produzidos em Minas Gerais, foram coletadas 24 amostras de soro de cada tipo de queijo (Minas padrão e mozarela) em quatro macrorregiões de Minas Gerais, em laticínios de médio e grande porte, sob inspeção federal. As amostras foram coletadas diretamente do tanque de produção, logo após o corte da massa, em recipiente plástico esterilizado acondicionado com gelo reciclável e transportado em caixas isotérmicas.

Recebido em 12 de maio de 2005

Aceito em 18 de dezembro de 2006

*Autor para correspondência (corresponding author)

E-mail: leorges@vet.ufmg.br

Apoio: FAPEMIG - Projeto CAG n.917/01 
O início das análises ocorreu em um prazo máximo de um dia após a coleta do soro, e foram realizadas análises de fosfatase alcalina e lactoperoxidase (Brasil, 2003) para verificação do emprego de pasteurização do leite utilizado.

As coletas foram realizadas durante o período de outubro (2002) a março (2003), relativo ao período chuvoso da região Sudeste, e o processamento do queijo de cada laticínio foi acompanhado para verificação do emprego das boas práticas de fabricação (BPF).

A análise microbiológica do soro consistiu nas pesquisas de coliformes (a 30 e a $45{ }^{\circ} \mathrm{C}$ ), psicrotróficos, bolores e leveduras e Staphylococcus coagulase positiva. Todas as técnicas utilizadas foram realizadas em duplicata e em conformidade com as normas para leite e derivados da International Dairy Federation (IDF) para cada microrganismo (IDF, 1985; IDF, 1997; IDF, 1990; IDF, 1991).
$\mathrm{O}$ experimento foi delineado como inteiramente ao acaso, com um fatorial $2 \times 4 \times 6$ (tipo de soro $\mathrm{x}$ região $\mathrm{x}$ repetições) (Sampaio, 2000). Para a comparação entre médias dos soros, utilizou-se o teste Mann e Whitney ( $\operatorname{programa~MINITAB}^{\circledR}$ ) e, para a comparação entre regiões, o teste Kruskal e Wallis (programa $\mathrm{SAEG}^{\circledR}$ ).

Os resultados microbiológicos (Tab. 1) em geral indicam que as BPF adotadas pelos laticínios não estão sendo efetivamente aplicadas. $\mathrm{O}$ fato de cerca de $90 \%$ das amostras de soro (exceto algumas oriundas da fabricação de mozarela) ter apresentado fosfatase alcalina inativada (negativa) indica pasteurização efetiva do leite usado como matéria prima. Isso sugere que a contaminação encontrada ocorreu durante o processamento, possivelmente por falha na higienização dos utensílios utilizados no processamento e dos próprios manipuladores. Segundo Souza et al. (1998), o manipulador é uma das vias mais freqüentes de contaminação dos alimentos.

Tabela 1. Contagem microbiana (\%) observada em soros de queijos minas padrão e mozarela

\begin{tabular}{lcc}
\hline Microrganismo & Soro de queijo mozarela & Soro de queijo-de-minas \\
\hline Coliformes a $30^{\circ} \mathrm{C}(\mathrm{NMP} / \mathrm{ml})$ & $4,1 \%<0,3$ & $4,1 \%<0,3$ \\
& $58,4 \%$ entre 0,4 e 150 & $58,4 \%$ entre 240 e 2,3 \\
Coliformes a $45^{\circ} \mathrm{C}(\mathrm{NMP} / \mathrm{ml})$ & $37,5 \% \geq 240$ & $37,5 \% \geq 240$ \\
& $12,5 \%<0,3$ & $29,1 \%<0,3$ \\
& $70,9 \%$ entre 1,1 e 110 & $58,4 \%$ entre 0,4 e 110 \\
Staphylococcus atípicos (UFC/ml) & $16,6 \% \geq 240$ & $12,5 \% \geq 240$ \\
& $4,2 \%<1,0 \times 10^{1}$ & $16,6 \%<1,0 \times 10^{2}$ \\
& $4,2 \%<1,0 \times 10^{2}$ & $75,0 \%$ entre $3,0 \times 10^{2}$ e $2,3 \times 10^{4}$ \\
Staphylococcus típicos (UFC/ml) & $75 \%$ entre $1,0 \times 10^{2}$ e $2,3 \times 10^{5}$ & $4,2 \% \geq 2,5 \times 10^{4}$ \\
& $16,6 \% \geq 2,5 \times 10^{5}$ & $4,2 \% \geq 2,5 \times 10^{5}$ \\
Staphylococcus coagulase positiva & $90,9 \%$ entre $9,0 \times 10^{1}$ e $2,7 \times 10^{3}$ & $50,0 \%=4,0 \times 10^{2}$ \\
(UFC/ml) & $9,1 \% \geq 2,5 \times 10^{4}$ & $50,0 \%=2,0 \times 10^{3}($ apenas 2 casos) \\
& $27,3 \%<1,0 \times 10^{1}$ & $50,0 \%<1,0 \times 10^{1}$ \\
Staphylococcus coagulase negativa & $13,6 \%<1,0 \times 10^{2}$ & $41,0 \%<1,0 \times 10^{2}$ \\
(UFC/ml) & $50,1 \%$ entre $1 \times 10^{2}$ e $1,5 \times 10^{4}$ & $4,5=4,7 \times 10^{3}$ \\
& $4,5 \%=1,8 \times 10^{5}$ & $4,5 \%=6,0 \times 10^{1}$ \\
& $4,5 \geq 2,5 \times 10^{6}$ & \\
Bolores e leveduras (UFC/ml) & $7,1 \%<1,0 \times 10^{1}$ & $11,1 \%<1,0 \times 10^{2}$ \\
& $7,1 \%=2,3 \times 10^{6}$ & $77,8 \%$ entre $1 \times 10^{3}$ e $2,1 \times 10^{4}$ \\
Psicrotróficos (UFC/ml) & $7,1 \%=7,0 \times 10^{6}$ & $11,1 \% \geq 2,5 \times 10^{6}$ \\
& $7,1 \% \geq 2,5 \times 10^{6}$ & \\
& $16,7 \%<1,0 \times 10^{1}$ & \\
& $70,9 \%$ entre $2,5 \times 10^{1}$ e $2,6 \times 10^{2}$ & $62,6 \%$ entre $2,0 \times 10^{1}$ e $1,7 \times 10^{3}$ \\
& $4,1 \% \geq 2,5 \times 10^{4}$ & $8,3 \% \geq 2,5 \times 10^{5}$ \\
& $8,3 \% \geq 2,5 \times 10^{5}$ & \\
& $25 \%<1,0 \times 10^{1}$ & $45,5 \%<1,0 \times 10^{1}$ \\
& $50 \%$ entre $1,4 \times 10^{2}$ e $4,0 \times 10^{2}$ & $32,9 \%$ entre $2,6 \times 10^{1}$ e $3,4 \times 10^{2}$ \\
& $25 \% \geq 2,5 \times 10^{5}$ & $4,5 \% \geq 2,5 \times 10^{3}$ \\
& & $4,5 \% \geq 2,5 \times 10^{4}$ \\
& & $13,6 \% \geq 2,5 \times 10^{5}$ \\
\hline
\end{tabular}

${ }^{1}$ Minas Padrão 


\section{Teixeira et al.}

De modo geral, não houve diferença estatística significativa entre tipos de soro, mas sim entre regiões, sendo que a região da Zona da Mata sempre apresentou as menores contagens microbianas e a região do Sul de Minas, as maiores. Na comparação entre os tipos de soros, somente os valores encontrados para Staphylococcus coagulase positiva e para colônias atípicas de Staphylococcus spp. apresentaram diferença significativa $(\mathrm{p}<0,01)$.

Em relação à contagem microbiana encontrada para coliformes a $30^{\circ} \mathrm{C}$ e a $45^{\circ} \mathrm{C}$, a porcentagem de amostras que apresentaram NMP/ml acima de 240 foi relevante, sendo que o soro de mozarela apresentou-se com maior contaminação. Estes dados são semelhantes aos encontrados por Chiappini et al. (1995a) e Siqueira (2000). A predominância dessa contaminação no soro de queijo mozarela pode ser atribuída ao hábito de alguns não pasteurizarem o leite para produção desse tipo de queijo. Além disso, as principais causas de contaminação por coliformes estão relacionadas à higiene tanto do processamento quanto do funcionário (Souza et al., 1998). Em relação a contaminação por Staphylococcus spp., as contagens encontradas nas maiores diluições, para ambos os soros, foi preocupante, tanto para colônias típicas quanto para colônias atípicas. É relevante a freqüência de colônias típicas coagulase positiva no soro de queijo mozarela, visto ser este microrganismo responsável por intoxicação por toxina estafilocócica, que é termorresistente. Contudo, também é relevante a ocorrência de Staphylococcus spp. de colônias coagulase negativa, pois estudos recentes têm demonstrado que essas estirpes também são capazes de produzir toxinas (Jay, 1993). Em relação aos bolores, leveduras e psicrotróficos, os soros apresentaram pouca contaminação por esses microrganismos, sendo que a contaminação foi mais expressiva no soro de mozarela. Estes resultados confirmam os encontrados por Siqueira (2000). No caso dos bolores e leveduras, a contaminação ambiental é o fator mais relevante. Ainda existe o fato de alguns microrganismos serem termodúricos, o que os torna resistentes à pasteurização, como é o caso de alguns psicrotróficos e micrococos. Isso ressalta a importância de uma matéria-prima de qualidade.

Os laticínios desta pesquisa são inspecionados pelo Serviço de Inspeção Federal e recebem leite de uma amostragem significativa do rebanho da região. Todos os estabelecimentos já haviam implantado ou estavam em processo de implantação das BPF, o que torna ainda mais preocupantes os índices de contaminação microbiológica encontrados. Os principais contaminantes microbiológicos encontrados foram coliformes e Staphylococcus spp., que são indicadores de higiene, destacando-se a região do Sul de Minas e o soro de queijo mozarela. Estes resultados ressaltam a necessidade da validação de técnicas para inspeção e da elaboração dos padrões de identidade e qualidade soro de queijo.

Palavras-chave: soro de queijo, mussarela, queijo-de-minas, perfil microbiológico

\section{ABSTRACT}

The microbiological profile of whey from the queijo-de-minas (Minas padrão) and mozzarella type cheeses produced in four of five most important regions of Minas Gerais state was studied. The contamination percentage found is serious, mainly in terms of the result found in coliforms count at $45^{\circ} \mathrm{C}$ e coagulase-positive Staphylococci. The contamination of the whey would be too high, indicating the material as unsuitable for human consumption as well as for industrial means. Due to the lack of a specific identity and a quality pattern for cheese whey, the results can be taken as a base for standard developments, which are seen as necessary concerning the microbiological quality of such products.

Keywords: cheese whey, mozzarella cheese, minas-padrão cheese, microbiological profile

\section{REFERÊNCIAS BIBLIOGRÁFICAS}

BRASIL. Ministério da Agricultura, Pecuária e Abastecimento (MAPA). Secretaria Nacional de Defesa Animal. Laboratório Nacional de
Referência Animal. Métodos Analiticos Oficiais para Controle de Produtos de Origem Animal e seus Ingredientes: II. Métodos físicos e químicos. Diário Oficial da União, n.83, p.15, seção 1, 2003. 
CHIAPPINI, C.C.J.; FRANCO, R.M.; OLIVEIRA, L.A.T. Avaliação do soro de queijo quanto aos coliformes totais e coliformes fecais. Rev. Inst. Lat. Cândido Tostes, v. 50, p.253-257, 1995a.

CHIAPPINI, C.C.J.; FRANCO, R.M.; OLIVEIRA, L.A.T. Avaliação do soro de queijo quanto à Staphylococcus aureus. Rev. Inst. Lat. Cândido Tostes, v. 50, p.259-263, 1995 b.

FURTADO, M.M.; LOURENÇO NETO, J.P.M. Tecnologia de queijos: manual técnico para a produção industrial de queijos. São Paulo:Dipemar, 1994. p.76-77.

MILK - IDF and milk based products: enumeration of coagulase-positive Staphylococci: colony count technique. In: Bulletin of International Dairy Federation, Brussels n. 145 A, p.5, 1997.

MILK - IDF and milk products: enumeration of coliforms: colony count technique and most probable number technique at $30^{\circ} \mathrm{C}$. In: Bulletin of International Dairy Federation, Brussels n.73 A, p.9, 1985.

MILK - IDF and milk products: enumeration of yeast e moulds: colony count technique at $25^{\circ} \mathrm{C}$. Bulletin of International Dairy Federation, Brussels, n. 94 B, p.2, 1990.

MILK - IDF and milk products: enumeration of psychrotrophic microorganisms: colony count technique at $6,5^{\circ} \mathrm{C}$. In: Bulletin of International Dairy Federation, Brussels, n. 101 A, p.3, 1991.

JAY, J.M. Microbiologia moderna de los alimentos. 3.ed. Zaragoza: Acribia, 1993. 804p.

KOSIKOWSKI, F.V. Whey utilization and whey products. J. Dairy Sci., v. 62, p.1149-1160, 1979.

MARTINS, E. Patrimônio de Minas. Jornal Estado de Minas, Belo Horizonte, dez, 2001. Caderno Economia, n. 44, p.14-17.

SAMPAIO, I.B.M. Estatística aplicada à experimentação animal. Belo Horizonte: Fundação de Ensino e Pesquisa em Medicina Veterinária e Zootecnia, 2002. 221p.

SIQUEIRA, I.M.C. Avaliação da qualidade físico-química e microbiológica de quatro tipos de soro de queijo. 2000. 104f. Dissertação (Mestrado) - Faculdade de Farmácia, Universidade Federal de Minas Gerais, Belo Horizonte.

SOUZA, C.M.; BRAGANÇA, M.G.; COLI, M.C.M. et al. Manual de boas práticas de fabricação de pão de queijo. Belo Horizonte: CETEC, 1998. 54p.

TEIXEIRA, L.V. Avaliação da qualidade físicoquímica e microbiológica do soro de queijos minas padrão e mussarela produzidos em quatro regiões de Minas Gerais. 2005. 42f. Dissertação (Mestrado) - Escola de Veterinária, Universidade Federal de Minas Gerais, Belo Horizonte. 